\title{
Acervo construido por futuros maestros de inglés y francés en su práctica en escuelas rurales ${ }^{1}$
}

\section{Foreign Languages Pre-service Teachers' knowledge Rooted in their Rural School Practicum}

\section{John Jairo Viáfara González ${ }^{2}$ Vitalia Pachón Achury ${ }^{3}$}

Citation/ Para citar este Artículo: Viáfara González, J. y Pachón Achury, V. (2021). Acervo construido por futuros maestros de inglés y francés en su práctica en escuelas rurales. Colomb. Appl. Linguistic. J., 23(1), pp. 35-48.

Received: 06-Jun.-2019 / Accepted: 15-Apr.-2021

DOI: https://doi.org/10.14483/22487085.16469

\section{Resumen}

Este estudio de caso cualitativo contó con la participación de futuros maestros de inglés y francés, hoy practicantes, pertenecientes a una escuela colombiana de idiomas que mantiene alianzas con instituciones educativas rurales de educación primaria en varias municipalidades vecinas. Mediante entrevistas y encuestas aplicadas a los participantes, los investigadores -también asesores- exploraron cómo las experiencias pedagógicas de estos practicantes en escuelas rurales influenciaron su construcción de conocimientos pedagógicos. Se determinó que la práctica en contextos rurales llevó a los futuros maestros a desmitificar principios pedagógicos para la enseñanza de lenguas previamente adquiridos, al considerar el propósito de los idiomas, la utilización de la lengua materna en las clases y la integración de temáticas tradicionalmente ajenas a la educación en esta área. Igualmente, se estableció que los participantes integraron teorías de pedagogía general en su labor, motivaron el desarrollo de habilidades interculturales en los niños y buscaron adaptarse a la metodología de la Escuela Nueva. Las implicaciones pedagógicas resaltaron la necesidad de incluir perspectivas socioculturales y críticas en el currículo de programas para la formación de futuros profesores de lenguas extranjeras.

Palabras clave: enseñanza de idiomas, Escuela Nueva, futuros profesores de lenguas extranjeras, lenguas extranjeras en educación rural, práctica pedagógica

\begin{abstract}
This qualitative case study included prospective English and French teachers. They were part of a Colombian School of languages which associated with primary rural schools at neighboring municipalities. Employing surveys and semi structured interviews, researchers explored how participants' pedagogical experiences at rural schools shaped their knowledge construction. The study found that pre-service teachers' practicums in these rural contexts allowed them to reexamine previously learnt foreign language teaching principles as they considered language use purpose, mother tongue use in classrooms and the integration of nontraditional topics in their teaching. Likewise,

1 Este artículo es producto de la investigación en curso titulada: "Foreign language pre-service teachers' pedagogical knowledge construction at rural schools", código (SGI 2535), realizada en el Centro de Investigaciones de la Facultad de Educación de la Universidad Pedagógica y Tecnológica de Colombia (UPTC).

2 Universidad Pedagógica y Tecnológica de Colombia (UPTC). ORCID (D): https://orcid.org/0000-0002-8409-6016. john.viafara@uptc.edu.co

3 Universidad Pedagógica y Tecnológica de Colombia (UPTC). ORCID (D): https://orcid.org/0000-0002-4547-0693. vitalia.pachon@uptc.edu.co
\end{abstract}


participants incorporated general pedagogy principles into their jobs, sought to encourage their students to develop intercultural abilities and to adapt their instructional practices to the Escuela Nueva approach. Pedagogical implications highlight the need to include socio-cultural and critical perspectives in foreign language pre-service teacher education curriculum development.

Keywords: foreign language teaching, Escuela Nueva, preservice foreign language teachers, foreign languages in rural education, teaching practice

\section{Introduction}

La exploración de la educación en lenguas extranjeras se ha enfocado, principalmente, en contextos urbanos, mientras que en las escuelas rurales es incipiente. En varios países las pocas investigaciones reportadas, centradas en el inglés, analizan el aprendizaje de este idioma mediado por factores geográficos, culturales, económicos, sociales, políticos y de recursos humanos (Arriagada, 2015; Chiu et al., 2013; Cruz-Arcila, 2018; Gil y Ruiz, 2009; González, 2010; Noguera, 2012; Olleta, 2013; Ortega y Fernández, 2017). Estos estudios han revelado la existencia de políticas educativas nacionales impuestas, homogeneizantes y, en gran medida, inoperantes para proveer los recursos y condiciones que aseguren una educación de calidad a los estudiantes y una educación continua y necesaria dirigida a profesores de países como Chile, España, Ecuador y Colombia.

Otros retos señalados por las investigaciones en México y Nicaragua tienen que ver con el paso de estudiantes de la escuela primaria a la secundaria, donde las exigencias de habilidades y conocimientos en lengua extranjera son mayores (Chiu et al., 2013; Noguera, 2012). De igual forma, son constantes las limitadas oportunidades de contar con recursos materiales y humanos apropiados (Chiu et al., 2013; Ortega y Fernández, 2017). Lo anterior conlleva al diagnóstico de que existen más condiciones desfavorables que favorables para el aprendizaje del inglés en contextos rurales.
En relación con los retos que los docentes enfrentan al involucrarse en la enseñanza del inglés en contextos rurales, se ha determinado que buena parte de las limitaciones se deben a la falta de oportunidades para que su propia educación sea eficiente y constante, de forma que los profesores puedan adaptar la enseñanza del idioma a los contextos en los cuales laboran y entenderlos, no exclusivamente en relación con la realidad sociocultural de sus estudiantes, sino también respecto a su propia identidad y saberes como maestros (Arriagada, 2015; Bonilla y Cruz-Arcila, 2014; Olleta, 2013; Ortega y Fernández, 2017).

Es por esto que el diagnóstico sobre las condiciones de maestros en ejercicio debe estar acompañado de acciones referentes a la educación de maestros en formación. Visto en el contexto colombiano, los estudios realizados por Quintero (2006), Ramos y Aguirre (2016), Rodríguez y Otálora (2018) y Varón et al. (2018) han detectado que los programas de formación inicial deberían educar a los futuros docentes para la enseñanza de idiomas en escuelas rurales.

Considerando el panorama anterior, el objetivo del presente estudio fue explorar el acervo de conocimientos pedagógicos que los participantes desarrollaron al momento de realizar sus prácticas en inglés y francés en escuelas rurales. En este sentido, se adoptó una perspectiva sociocultural para abordar la pregunta base de la investigación: ¿qué conocimientos referentes a los procesos pedagógicos que subyacen a la enseñanza y el aprendizaje del inglés y el francés construyen los participantes en el marco de sus prácticas docentes en escuelas rurales de educación primaria?

\section{Marco Teórico}

Esta sección discute la teoría sociocultural enfocada en la construcción de conocimientos por parte de los maestros, revisa conceptos respecto a la ruralidad y la educación, y finalmente incluye un resumen de hallazgos relevantes en estudios nacionales e internacionales sobre la enseñanza de lengua extranjera en contextos rurales. 


\section{La base de conocimiento que construyen los maestros: una perspectiva sociocultural}

Históricamente las teorías que buscan describir las bases de conocimientos que se deberían construir para llevar a cabo la labor docente tienden a emplearse indistintamente de los sujetos, sean docentes en ejercicio o en formación. Dos teorías han tenido una notable influencia en el área de aprendizaje de lenguas extranjeras. La primera, postulada por Shulman (1987) y procedente de una perspectiva general de la pedagogía, ha sido cuestionada por la segunda, propuesta por Freeman y Johnson (1998) en el campo de las lenguas segundas o extranjeras. Estos últimos critican el carácter atomizante de la teoría de Shulman (1987), pues destacan, entre otras limitaciones, su desconocimiento acerca de las circunstancias que afectan el quehacer de los docentes. En contraste, proponen una perspectiva sociocultural basada en tres dimensiones interrelacionadas (Freeman y Johnson, 1998).

En primer lugar, los docentes en formación o ejercicio son vistos como individuos involucrados en un proceso constante de autoconstrucción y reformulación de sus saberes. Este proceso implica la propia comprensión de los factores múltiples que pueden influir en su aprendizaje como profesores. En segundo lugar, se espera que ellos desarrollen un conocimiento profundo respecto a la naturaleza del proceso pedagógico que subyace tras la enseñanza y el aprendizaje de las lenguas extranjeras. Dicho saber también ha sido llamado "conocimiento pedagógico del contenido" (Shulman, 1987). Finalmente, los profesores o futuros maestros necesitan desarrollar saberes relacionados con las características políticas, históricas, sociales y culturales de los ámbitos educativos donde se emplean. Es decir, como menciona Freeman (2002), las escuelas pueden mediar en la elaboración y reconstrucción que los docentes hagan de aquello que aprenden y la forma en que lo interiorizan.

Bajo una perspectiva Vigotskiana, Johnson (2009) recalca que el aprendizaje de los maestros de idiomas es una labor de carácter social, realizada en el marco de situaciones sociales y relacionada con los individuos y con las tareas y herramientas que estos emplean y utilizan. Es de esta manera que la perspectiva sociocultural contribuye a comprender la función que las interacciones sociales cumplen en los procesos de pensamiento en los cuales se involucran los docentes cuando aprenden cómo enseñar.

En conclusión, se puede afirmar que los maestros hacen de sus saberes y habilidades una práctica social con sentido justo en el momento en que se relacionan con un contexto específico, y que (dicha práctica) abarca procesos discursivos, de mediación, de interacción social y de estructuras de participación. Esta elaboración de conocimientos es simultánea, como lo discuten Singh y Richards (2006), y arraigada tanto a las ideologías de los profesores mismos como a sus escuelas para reflejar su comprensión de lo que significa, o debería significar, el aprendizaje. Entre tanto, Burns y Richards (2009) manifiestan que el conocimiento de los profesores tiene un carácter cambiante, pues depende del énfasis que hagan los maestros tanto en sus necesidades como en los intereses de la comunidad a la cual pertenecen.

\section{Ruralidad y educación}

Sin desconocer perspectivas actuales de lo que constituye la ruralidad, las cuales reconocen el impacto del contexto urbano en el rural (Zamora et al., 2012), y considerando la caracterización que investigadores nacionales e internacionales han hecho de la citada ruralidad, el presente estudio entiende que los contextos rurales no son totalmente similares; en general, la población en estas zonas es menor que en las áreas urbanas y no existe una concentración de personas en zonas específicas, pues la población tiende a estar dispersa (González, 2010; Olleta, 2013; Perfetti, 2004). Por lo regular, debido a que no hay suficientes medios de transporte masivo disponibles para la población, las distancias entre municipalidades pueden ser extensas (Olleta, 2013). González (2010) explica que las condiciones de las vías para transporte son deficientes en muchos casos, comparadas con las áreas urbanas, y por lo general la población rural tiene a su disposición menos servicios sociales y de calidad deficiente. Perfetti (2004) subraya que 
la pobreza es mayor y que es más complicado el manejo de la educación, gestionada -casi en su totalidad-desde el sector público.

A partir de estudios realizados por González (2010), Martínez et al. (2016) y Perfetti (2004) se ha determinado que las instituciones educativas en los contextos rurales presentan en muchos casos limitaciones en infraestructura y en recursos. Igualmente, como lo mencionan Martínez et al. (2016), en estos contextos es habitual la heterogeneidad en relación con las condiciones socioeconómicas y las características personales de los estudiantes. En particular, en la educación primaria, los investigadores mencionados al principio del párrafo señalan que, debido a las distancias extensas entre las escuelas y los hogares, el trabajo agrícola de los niños y la escasa educación de sus padres o cuidadores, los índices de retención son más bajos.

Históricamente, el Ministerio de Educación Nacional (MEN) ha impulsado la implementación de modelos educativos acordes con las especificidades del ámbito rural (Martínez et al., 2016). Uno de estos modelos, Escuela Nueva, iniciado en Colombia en la década de los 70, tiene como fundamento una perspectiva multigrado en la cual los profesores actúan como guías enseñando a niños de diferentes edades simultáneamente; con ello se espera que los estudiantes sean más autónomos, en contraste con aquellos inscritos en instituciones tradicionales. Asimismo, Gómez (2010) indica que, en este enfoque, el uso de estrategias y recursos, como guías de autoaprendizaje, bibliotecas y esquinas de aprendizaje, no solo motivan la independencia, también fomentan el aprendizaje activo, colaborativo y comunitario.

\section{La escuela rural y los docentes de lengua extranjera}

Teniendo en cuenta que este estudio se centra en futuros maestros de lenguas extranjeras en contextos rurales, y debido a la escasez de estudios sobre estos profesionales, la presente sección incluirá hallazgos referentes a profesores en ejercicio. En general, las investigaciones publicadas que involucran docentes en ejercicio en contextos rurales dan cuenta exclusivamente de la enseñanza del inglés y no del francés. Los docentes, usualmente, no son especialistas en la enseñanza del inglés, pues no poseen diplomas en esta especialidad (Arriagada, 2015; Olleta, 2013). No obstante, lo asumen como parte de su labor, la cual incluye la instrucción en múltiples áreas del conocimiento. Lo anterior indica, como lo muestran los estudios realizados por Arriagada (2015), Cruz-Arcila (2018), Noguera (2012), Olleta (2013), Ortega y Fernández (2017), Roldán y Peláez (2017) y Varón et al. (2018), que las condiciones para la formación de los docentes en lengua extranjera son precarias y los recursos materiales, en muchos casos, son inapropiados (Chiu et al., 2013; Ortega y Fernández, 2017). Este panorama se hace aún más complejo al observar, a partir de los trabajos arriba citados, que este reto lo afrontan docentes de varios países hispanohablantes como Chile, España, Ecuador, México y Colombia.

En lo referente a la heterogeneidad de estudiantes en aulas multigrado, Olleta (2013) señala una preocupación respecto a los textos utilizados en clase, pues en gran medida se fundamentan en los niveles de habilidad de los estudiantes, y los maestros tienden a recurrir a la generalización del contenido; por tanto, con su uso se desconoce la diversidad. Igualmente, el uso de metodologías tradicionales que no motivan a los estudiantes y la baja promoción de interacción en el idioma han sido señalados como desafíos por Gil y Ruiz (2009) y Rodríguez y Otálora (2018). Ahora, mientras que el bajo aprovechamiento del contexto sociocultural para la instrucción de los estudiantes se percibe como una problemática en el estudio de Gil y Ruiz (2009), en el trabajo de Cruz-Arcila (2018) se resalta el esfuerzo de los maestros por lograr una coherencia entre sus saberes y acciones cuando realizan su labor en conexión con el contexto rural y la metodología de la Escuela Nueva en Colombia. Desde la perspectiva de Bonilla y Cruz-Arcila (2014), los retos anteriores generan preocupación por su efecto para el desarrollo de conocimientos y habilidades en los estudiantes, así como por la inseguridad que presentan los profesores para trabajar en las clases. 
En relación al conocimiento que futuros maestros construyen mientras realizan sus prácticas pedagógicas en contextos rurales colombianos, encontramos dos investigaciones: por un lado, Quintero (2006, p. 158) realizó un estudio de caso cualitativo basado en "diarios de campo, observación directa, entrevistas cualitativas, filmaciones, grabaciones y registros", en el que involucró a 10 estudiantes en su último año de licenciatura. Por su parte, Zuluaga et al. (2009) condujo una investigación acción por medio de observaciones, diálogos informales y revisiones de documentos que contó con supervisores de práctica, maestros en formación y estudiantes de tres escuelas. Los dos estudios tuvieron como escenario la zona cafetera y establecieron que, a través de esta experiencia, los participantes desarrollaron un conocimiento pedagógico anclado en la conciencia cultural local. En ambos hubo un impacto curricular significativo, orquestado por los futuros maestros, quienes integraron lengua y contenido, y diseñaron materiales relevantes para la enseñanza del inglés. Finalmente, Zuluaga et al. (2009) resaltaron la creciente sensibilidad de los participantes hacia las problemáticas regionales, familiares e individuales de los estudiantes, y la promoción de transformación en las aulas, con miras a mejorar los procesos pedagógicos.

\section{Metodología}

Al optar por un estudio de caso cualitativo (Yin, 2013), en esta investigación se buscó conservar lo holístico y el sentido característico de los eventos de la vida real. Así mismo, se exploraron contextos reales, como la práctica pedagógica en inglés y francés en escuelas rurales, teniendo como unidad (Merriam, 2009; Yin, 2013) a un grupo de futuros docentes de lenguas extranjeras de una universidad pública del centro de Colombia. Además, cabe resaltar que este enfoque estuvo alineado con la perspectiva sociocultural anteriormente discutida, pues permitió considerar los factores situacionales que interactuaron alrededor del caso investigado para así comprender el desarrollo del conocimiento pedagógico por parte de los participantes.

\section{Participantes y contexto}

Este estudio contó con la participación de 68 maestros en formación de la Escuela de Idiomas de la Universidad Pedagógica y Tecnológica de Colombia (UPTC). El 70\% de ellos pertenecientes a la Licenciatura en Lenguas Extranjeras (inglés y francés) y el $30 \%$ a la Licenciatura en Idiomas Modernos (español e inglés). La Tabla 1 relaciona el número de estudiantes participantes por programa, los cursos en los cuales realizaron las prácticas, el semestre cursado de acuerdo al plan de estudios, los idiomas enseñados, y las horas y semanas de práctica en instituciones.

Los participantes se ubicaron en un estrato socioeconómico 2 y 3 , y sus lugares de origen incluían municipios y ciudades de los departamentos de Boyacá, los Santanderes, Cundinamarca y los Llanos Orientales. Las edades de las 46 maestras y 22 maestros en formación oscilaron entre los 19 y 25 años. Los dos investigadores responsables de este estudio fueron también asesores de práctica docente de los participantes.

Tabla 1. Participantes y contexto del estudio investigativo.

\begin{tabular}{|c|c|c|c|c|c|c|}
\hline \multirow{2}{*}{ Programa } & \# Participantes & Cursos & $\begin{array}{c}\text { Lengua(s) } \\
\text { enseñada(s) }\end{array}$ & Semestre & \multicolumn{2}{|c|}{ En Instituciones Educativas } \\
\cline { 3 - 7 } & 18 & Didáctica I y II & Inglés & VII y VIII & 2 & 10 \\
\hline $\begin{array}{c}\text { Idiomas } \\
\text { Modernos }\end{array}$ & 9 & Práctica II & Inglés & IX & 4 & 10 \\
\hline $\begin{array}{c}\text { Lenguas } \\
\text { Extranjeras }\end{array}$ & 41 & Práctica Final & Inglés y Francés & X & 12 & 14 \\
\cline { 2 - 7 }
\end{tabular}

Fuente: elaboración propia. 
Las prácticas se llevaron a cabo en varios municipios del centro de Boyacá conocidos por su geografía -rica en recursos naturales-, cuyas actividades económicas, especialmente en zonas rurales, están basadas principalmente en la agricultura y la ganadería. En la Figura 1 se detallan los municipios donde los participantes realizaron sus prácticas.

\section{Recolección de datos}

Los datos se reunieron gracias a la aplicación de una encuesta en línea que, de acuerdo a Cohen et al. (2018, p. 471), es un instrumento que provee información estructurada, fácil de administrar sin la presencia del investigador y relativamente sencilla de analizar. El instrumento fue aplicado a 68 participantes y exploró, en primera medida, sus antecedentes en relación a la educación recibida -primaria y secundaria- con el fin de entender la experiencia de vida que tuvieron como aprendices de una lengua extranjera. Luego se indagó por la construcción de conocimiento de los participantes en relación con la práctica rural en inglés y francés: se incluyeron preguntas cerradas acerca del perfil de los participantes y sus asignaciones de práctica, que ayudaron a describir el contexto de estudio. Respecto a los datos -los porcentajes presentados anteriormente-, estos fueron analizados con la ayuda de la estadística descriptiva (Dörnyei y Taguchi, 2009). Así mismo, las preguntas abiertas exploraron las percepciones de los participantes, pero también la información factual basada en sus narraciones a partir de sus experiencias pedagógicas. El instrumento fue aplicado inicialmente a un grupo de 10 estudiantes que ya habían realizado la práctica pedagógica; a partir de sus respuestas se ajustó, y finalmente fue administrado (al resto) mediante la aplicación de internet: Google Forms.

Como segunda medida se recolectaron datos por medio de una entrevista semiestructurada a 26 participantes voluntarios. Dieciocho (18) fueron presenciales y ocho (8) virtuales (vía Skype y WhatsApp); cada una duró aproximadamente 45 minutos y fue grabada mediante teléfono celular y grabadora digital. Se incluyeron preguntas preparadas con antelación y otras a partir de las respuestas de los participantes. Como lo menciona Berner-Rodoreda et al. (2020), con este instrumento se buscó obtener información del entrevistado para profundizar en la comprensión del tema. Se comenzó con preguntas tendientes a construir un perfil del participante como aprendiz de lenguas extranjeras, posteriormente se interrogó en relación con la pregunta que guía el estudio y finalmente se indagó respecto a las respuestas de los futuros maestros en las encuestas.

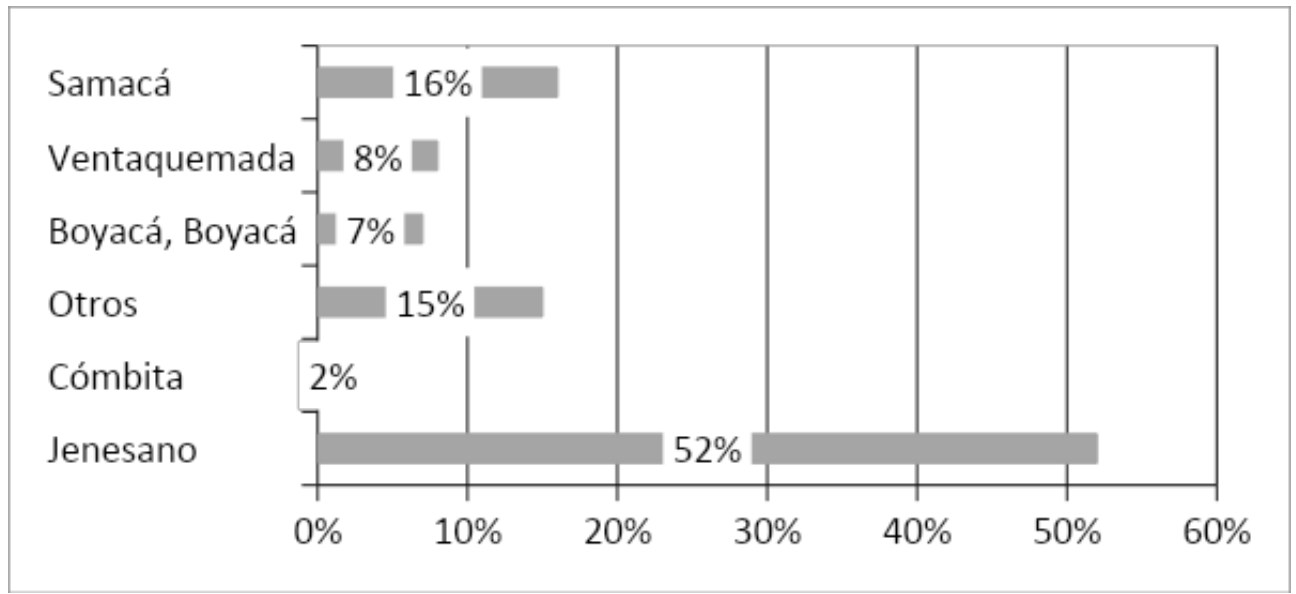

Figura 1. Distribución de prácticas en municipios boyacenses.

Fuente: elaboración propia. 


\section{Hallazgos y Discusión}

El estudio de los datos se realizó bajo el enfoque de análisis temático, el cual implica codificar los datos y, posteriormente, organizarlos en grupos de códigos que comparten similitudes por temas (Glesne, 2015). El software Atlas ti facilitó la clasificación, exploración, visualización y codificación de la información en encuestas y entrevistas siguiendo la metodología propuesta por Miles et al. (2014). En primer lugar, se identificaron patrones comunes en los datos relevantes. Luego, estos códigos iniciales se agruparon con otros similares a fin de reducir su número, lo que dio lugar a códigos macros y, finalmente, a las categorías y subcategorías (temas y sub-temas) que responden a la pregunta de investigación.

El estudio también siguió los principios de triangulación metodológica y de investigadores, y empleó la validación por participante "member checking" (Merriam, 2009). Dieciséis (16) participantes respondieron voluntariamente a la invitación para la validación y todos corroboraron que los hallazgos eran fieles a sus experiencias y percepciones respecto a la construcción de un conocimiento pedagógico. A continuación, se discute el conocimiento sobre la enseñanza de los idiomas que los participantes manifestaron haber desarrollado en sus prácticas rurales.

\section{Prácticas en contextos rurales y la desmitificación de la pedagogía para la enseñanza de las lenguas}

Las inquietudes y los requerimientos que el contexto rural planteaba a los futuros maestros ocasionaban que estos actuaran guiados por una reconceptualización de saberes, originalmente construidos en las aulas universitarias y mediados por académicos estudiados -sus maestros o compañeros-. Esta desmitificación de aspectos pedagógicos puede explicarse, como lo discuten Freeman (2002), Freeman y Johnson (1998) y Singh y Richards (2006), por la asociación que surge entre la influencia del contacto de los participantes con las prácticas, creencias, valores, objetos y personas en el contexto sociocultural escolar, y la formación de sus saberes. Estudios realizados por Bonilla y
Cruz-Arcila (2014) y Cruz-Arcila (2018) demuestran cómo el aprendizaje de docentes en ejercicio, en contextos rurales, privilegia -en muchos casossus propias teorías por encima de lo aprendido en contextos formales de educación, condición que parece alinearse con el caso de los profesores en formación que se discute a continuación.

El primer tema común entre los participantes se refiere a la comprensión significativa respecto a si el propósito primordial de utilizar los idiomas es la comunicación. Según sus testimonios, orientar la enseñanza hacia la función comunicativa de las lenguas les permitió guiar a sus estudiantes desde el comienzo de la clase, evitar la desmotivación y la indisciplina, ofrecer oportunidades para estimular la producción oral y generar relaciones interpersonales positivas con los niños. Sobre esto, una participante comentó:

Yo creo que la idea que le dejé a esos chicos es que el aula de clase tiene que ser para la comunicación [...]. Les ponía pequeños retos: tenían que encontrar, por ejemplo, quién había secuestrado el gato y en qué parte de la casa. Así, mediante preguntas, los empujaba a hablar y los tenía muy motivados (P25E)4.

Además de tareas como la descrita anteriormente en una clase de francés, los participantes motivaron a sus estudiantes a asumir actividades de liderazgo dentro del aula. Allí, fue habitual conectar las temáticas estudiadas con la vida cotidiana de los niños. Como consecuencia, los maestros practicantes se sorprendieron al escucharlos emplear lo aprendido en contextos fuera del aula, como en los comedores, el camino hacia la escuela o en sus hogares. Así lo manifestaron los padres de familia:

Cómo sería de chévere que hasta jugaban la golosa y contaban en francés. Llegaban los padres de familia a dejarlos y me decían: "uy no profe, eso llegan y me dicen un montón de cosas que yo no entiendo nada, pero suena bien bonito" (P26Q).

4 Los códigos al final de los testimonios indican el participante $(\mathrm{P})$, identificado con un número, y el instrumento del cual se extrajo el testimonio (E, entrevista; $Q$, cuestionario). 
Dar prioridad al propósito comunicativo de los idiomas implicó también que el español pasara de ser considerado un villano a ser visto como un aliado en el aprendizaje del inglés y el francés. La timidez y la falta de exposición a la lengua extranjera, que según los futuros docentes caracterizaban a muchos de los participantes, complicaban el empleo de estos idiomas como medio privilegiado de interacción en el aula, causando que en su espontaneidad los niños recurrieran al español, lo combinaran con la lengua extranjera o sintieran la necesidad de traducir cada palabra dicha por el futuro docente:

Los estudiantes en clase, incluso con el lenguaje transparente, no entendían. Y en la universidad a uno le decían que la traducción no era algo muy bueno. Pero llega el momento en que uno dice 'no más, pues digamos la palabra en español a ver si de una u otra manera a los niños se les queda'. A mí me funcionó y a mis compañeros de trabajo también, hablamos y encontrábamos eso en común (P38E).

Publicaciones en el ámbito nacional e internacional mencionan cómo estudiantes practicantes utilizan su lengua materna con el fin de apoyar y monitorear la comprensión, y construir significado en contextos específicos de enseñanza (Viáfara, 2011). Autores como Hittotuzi (2006), considerando factores socioculturales y pedagógicos, discuten el papel de la lengua materna en la enseñanza de idiomas extranjeros.

No obstante, el uso del español presentó varios retos: por un lado, los maestros practicantes se vieron enfrentados a una variedad de español distinta a la que ellos empleaban, al provenir de un contexto citadino y universitario. Los términos utilizados por los niños para denotar en lengua materna los nombres de objetos, lugares, animales y plantas, entre varios, eran diferentes en ciertas ocasiones. $\mathrm{Y}$ algunos profesores practicantes instruyeron a los niños en el uso "correcto" del español de acuerdo con la variedad socialmente privilegiada que ellos traían. Sin embargo, esta labor pedagógica, que pretendía aliviar impases al usar el español como lengua franca, al basarse en una imagen negativa de la variedad utilizada por los niños, termina avalando el imperialismo lingüístico del español respaldado por la academia sobre la variedad de español rural utilizada por los estudiantes y, en consecuencia, deslegitima el valor identitario del español rural a partir de ideologías lingüísticas puristas:

Hay mucho vocabulario que ellos [los niños] no conocen, incluso en español, pues obviamente es distinto. Tenemos que corregir muchísimo, bien sea por cultura, el lenguaje que utilizan, pues todo es aprendizaje y es importante que ellos aprendan a hablar bien español. No hay que tener temor en decirles: "chicos, no se dice de esa manera, se dice así" (P27E).

Los futuros maestros también reevaluaron los conocimientos y habilidades que son prioritarios para apoyar el aprendizaje de los niños en el contexto rural y se centraron en lo que se mostraba relevante en este. Retaron la creencia de que la enseñanza de vocabulario es lo primordial al enseñar lenguas extranjeras a infantes. Es más, retaron lo que consideraban un principio plantado desde su educación universitaria: "Que el idioma extranjero sólo debe enseñárseles a los niños de manera oral" (P5E), como lo explica esta participante, y continúa: "Aunque esto sea en parte verdad, a los niños también podemos introducirles lecturas simples pero didactizadas, con muchas imágenes $y$, obviamente, buscando el momento correcto, de la misma manera que hacemos con la escritura." Las experiencias previas de los niños en este contexto particular en el cual han sido educados, generalmente, por maestros que, al no tener un manejo fluido del idioma oral, optan realizar la mayoría del trabajo de forma escrita (esta modalidad también es la preferida por los estudiantes), guiaron el replanteamiento metodológico de los futuros maestros, como lo muestra el siguiente fragmento:

Ellos trabajaban la escritura antes y no tenían casi contacto oral con la lengua. Yo llegué trabajando la parte oral, pero después me di cuenta de que a ellos les gustaba mucho escribir porque estaban acostumbrados a eso anteriormente [...] decidimos [con mi compañero], entonces, dividir el plan de clase en dos: en la primera parte trabajar mucho 'listening' y en la segunda la parte escrita. Yo antes creía que la escritura no debía hacerse y más viendo que ya habían 
trabajado mucha la escritura. Por eso les llevé un libro, y ahí notamos que les gustaba mucho mirar las letras y mirar las imágenes, y relacionarlas (P6E).

El subtema final involucró el contacto con las tradiciones y las creencias de la cultura local frente a las cuales, en ocasiones, los participantes entraron en conflicto al tratar de enseñar las lenguas extranjeras. Las creencias y prácticas religiosas, los estereotipos con respecto a lo convencional -o no- en asuntos de género, las visiones sobre las instituciones sociales como la familia y las opiniones alrededor de ciertas prácticas, como el consumo de licor, se convirtieron en material significativo para la labor pedagógica. Estas temáticas, que en muchos casos parecieran ser ajenas a las tendencias comunes en el aula de lengua extranjera y más aún en la enseñanza a infantes, fueron desmitificadas por los futuros maestros, quienes, de esta forma, integraron una perspectiva crítica, reconocieron la substancia que compone el contexto escolar y actuaron acorde con él; además, le dieron forma a las posibilidades de aprendizaje desde el aula que podían permear la vida de sus estudiantes. A pesar de que en algunas ocasiones irrumpir en la cultura local trayendo diferentes ópticas de ver el mundo causó temor a los participantes y algunas tensiones con los padres de familia, los maestros en formación se aventuraron a aprovechar temáticas, como las mencionadas anteriormente:

Un día llegó un policía a dar unas indicaciones para la época de navidad respecto a el consumo de estas sustancias [alcohol y tabaco], y los niños estaban muy interesados en saber sobre esto. Después de la charla ellos [los niños] hablaron sobre cuáles familiares en sus casas fumaban mucho y bebían, y yo les empecé a explicar algunos efectos de esas sustancias y por qué era malo para el cuerpo consumirlas. Ahí les recordé algún vocabulario ya visto, las partes de la cara (dientes amarillos), por ejemplo, para retratar las consecuencias (P5Q).

Los datos recolectados revelan instancias en las cuales los maestros en formación articularon el proceso de enseñanza-aprendizaje con el ámbito sociocultural en el que trabajaron, al comprender integralmente el contexto escolar y construir una pedagogía significativa que se optimiza en la medida en que estas situaciones también mejoran (Kumaravadivelu, 2006). Los participantes también manifestaron interés por lograr construir más oportunidades de bienestar para sus estudiantes en comunidades rurales, fomentando en ellos una mentalidad más abierta que les permita interactuar con personas fuera de su contexto inmediato y construir, desde edades tempranas, actitudes de cuidado hacia sí mismos (Hawkins, 2004). Otros estudios en contextos rurales colombianos(Quintero, 2006; Zuluaga et al., 2009) reportan cómo futuros maestros configuran opciones metodológicas que superan los modelos descontextualizados y aprendidos durante su formación académica.

\section{Principios de Escuela Nueva en la enseñanza de lenguas extranjeras}

Los participantes se esforzaron por articular su conocimiento y habilidad pedagógica con el contexto educativo de la Escuela Nueva. Para ellos, este enfoque fue algo totalmente novedoso o que habían conocido de manera teórica en la universidad. En sus testimonios, los futuros maestros dejan ver la necesidad de recurrir a su capacidad de análisis, creatividad y recursividad para manejar apropiadamente el reto que esta articulación implicó. De igual manera, señalaron a sus pares y profesores titulares como apoyos importantes para trabajar desde este enfoque. En relación con estos últimos, recalcaron su papel primordial como modeladores de ideas que luego fueron analizadas y poco a poco optimizadas por los futuros maestros.

Los participantes señalaron como la mayor ganancia de su inmersión en la pedagogía de la Escuela Nueva su incremento en habilidades para trabajar con estudiantes de diferentes grados, edades y, por ende, capacidades, ritmos de aprendizaje, intereses y necesidades. En este sentido, aprendieron "cómo manejar las diferencias", "que no podían esperar lo mismo de todos los estudiantes" y "lo importante que fue apoyar a los niños para que ellos aprendieran a 'educarse a sí mismos'”. Al enfrentarse al reto de las aulas multigrados, fortalecieron su planeación, 
teniendo en cuenta la variación y la inclusión de actividades y materiales significativos, junto con el manejo de la clase (organización de los estudiantes en el aula y fomento de valores):

Creo que es un desafío enfrentar un salón de clases con niños de diferentes edades y, así mismo, preparar un plan de clase para todos. Lo mejor es reconocer su nivel y hacer uso de diferentes estilos de aprendizaje y de todos los recursos que ellos tengan al alcance, pues son personas que quieren saber más del mundo (P45Q).

Los maestros practicantes reconocieron y valoraron el trabajo colaborativo como una estrategia para enfrentar la marcada diversidad en la organización de las aulas en la Escuela Nueva: "para lidiar con estos tres niveles diferentes el trabajo colaborativo es muy efectivo, las profesoras lo implementaron con estudiantes del mismo grado, pero entre todos los grados diferentes no lo vi, aunque me funcionó cuando lo implementé (P25E)". De igual manera, comprendieron el valor que tiene fomentar la autonomía en los estudiantes desde edades tempranas: "Tienen que ser muy autónomos, muy responsables para trabajar y aportar al grupo [...] ya sabían que tenían que esperar, que si no entendían le preguntaban a otros compañeritos que sí entendían mientras yo llegaba, y eso fomenta orden (P25E)". La autonomía, que los futuros maestros con frecuencia asociaban a la responsabilidad que muchos niños adquirían desde temprana edad por su trabajo en el campo, no únicamente la advirtieron en su capacidad de autorregulación para abordar el propio aprendizaje en el aula, sino también en la capacidad propositiva de los infantes.

Aun así, los esfuerzos de los futuros maestros por integrar el enfoque de la Escuela Nueva a la enseñanza del inglés y el francés no fue siempre un trabajo fácil:

La experiencia que más me marcó fue enseñar a niños de preescolar en el mismo salón de niños de primero y tercero. Fue muy complicado encontrar estrategias que captaran la atención de los niños de preescolar. La enseñanza del inglés fue muy fragmentada debido a la brecha de saberes y el poco tiempo para guiarlos (P3Q).

Este hallazgo se relaciona con investigaciones que subrayan las dificultades que la clase multigrado puede implicar para los docentes en el proceso de enseñanza del inglés (Olleta, 2013) y la falta de conocimiento que algunas veces los maestros demuestran al trabajar en contextos rurales (Quintero, 2006; Rodríguez y Otálora, 2018; Varón et al., 2018), pues los maestros colombianos son educados con frecuencia para enseñar a poblaciones homogéneas en contextos urbanos.

\section{Pedagogía general y enseñanza de las lenguas extranjeras}

Los maestros en formación incrementaron su comprensión sobre algunos aspectos generales de la pedagogía, ligados a la enseñanza de las lenguas extranjeras en este contexto rural. Reportaron que los niños se interesaban más en el aprendizaje cuando se trataba de temas que tuvieran relación con su entorno y sus costumbres: "los estudiantes tienen intereses como su familia y las labores familiares, el campo y las actividades agrícolasescolares. Todo esto puede ser usado como punto de referencia en la enseñanza y aprendizaje de la lengua extranjera (P52Q)".

Como lo señala Pérez (2016), el sistema educativo no debe servir únicamente para preparar y capacitar a los alumnos en el dominio de ciertas habilidades cognitivas y técnicas instrumentales, también debe formar ciudadanos críticos $\mathrm{y}$ comprometidos con su realidad social y cultural, es decir, como miembros activos y responsables de su sociedad, su cultura y su mundo.

El interés de los niños en asignaturas cercanas a su diario vivir llevó a los maestros en formación a tener en cuenta la transversalidad curricular, incluyendo temáticas de áreas como la ecología, el cuidado del medio ambiente, las ciencias sociales y cívicas, entre otras, en la clase de lengua extranjera. Así, los niños pudieron hablar y realizar actividades de su interés mediante el uso del inglés y el francés, como lo señaló un participante: "Conocer las plantas y las semillas del lugar, y trabajar con ello en el 
aprendizaje de la lengua extranjera fue un buen elemento [...] los niños llevaban sus herramientas y mientras sembraban iban preguntando cómo se decía cada elemento o acción en el idioma extranjero (P26Q)".

Por otra parte, el trabajo con niños de necesidades especiales, principalmente las concernientes a discapacidad física, trastornos del espectro autista, trastornos de déficit de atención y de conducta, y problemas de aprendizaje, significó la construcción de nuevos conocimientos. La inclusión supuso un reto adicional para los futuros docentes, quienes - de cierta manera- buscaron estrategias que integraran a todos los estudiantes dentro de sus clases:

\section{[...] había un estudiante con Síndrome de Down. Este fue un gran reto para mí, puesto que el niño no podía seguir mis clases tal como lo hacian sus compañeros. Por eso decidí prestar atención a sus necesidades y ver de qué manera podía llegar hasta él [...] pude ver que le gustaban mucho las artes $y$, en especial, pintar, así que de esa manera traté de acercarme y enseñarle (P5Q).}

Los maestros en formación coincidieron en que, en contextos rurales, existe una falta de presencia y apoyo de expertos que traten estas necesidades especiales, e incluso los docentes titulares no cuentan con ese tipo de acompañamiento. Sanz del Río (1991) señala que se hace necesaria la integración de los profesores especialistas en estos temas y los profesores de clases ordinarias, pero en las zonas rurales es muy escasa la presencia de los especialistas debido a las largas distancias y a sus asignaciones, que privilegian las zonas urbanas.

Finalmente, según los testimonios de los futuros docentes, podemos establecer que ellos desarrollaron una comprensión de la dimensión intercultural durante su labor pedagógica. Dos vértices fueron privilegiados por los participantes al aproximar el componente intercultural a su pedagogía: la cultura urbana y la cultura rural; y la cultura de la lengua extranjera y la colombiana. Fue así como la clase de inglés y francés se convirtió en un espacio para intercambiar ideas con respecto a estos ámbitos. En relación con los contextos urbano y rural, un participante mencionó:

Preparé mi clase sobre las profesiones que
normalmente uno ve en ciudad (policía,
doctor, profesor, futbolista) y les pregunté a
los estudiantes qué querían ser cuando fueran
grandes. Las respuestas fueron: granjero,
vendedora, soldado, tractorista, volquetero,
entre otros. Ahí me di cuenta de que, así como
nosotros aprendemos mucho de los niños en
estos contextos, también podemos ser el canal
perfecto para que ellos conozcan el mundo
urbano de una buena manera (P63E).

En concordancia con lo expresado por este participante, Paige et al. (2000) señalan que, según Byram (1991), los maestros (participantes de un estudio investigativo) hablaron sobre la importancia de que los niños conocieran otras formas de vivir, debido a que este conocimiento les permite ser tolerantes con los demás y menos restringidos con su propio estilo de vida. Esto genera una conciencia cultural, centrándose en la cuestión de cambio de la competencia monocultural a la intercultural.

Respecto al segundo vértice, los futuros docentes lograron trabajar aspectos de la cultura extranjera relacionándola con la colombiana para motivar y despertar en los niños el deseo de seguir aprendiendo las lenguas extranjeras y la importancia de hacerlo:

Yo pensé que una manera de motivarlos a ver la importancia del inglés era mencionar los avances que ha tenido el sector agrícola en los Estados Unidos. Así que contándoles cómo les beneficiaba el conocimiento de una lengua extranjera en toda actividad, pude fortalecer el apoyo familiar de los padres a mis estudiantes $y$ elevar las expectativas con respecto a continuar aprendiendo una segunda lengua (P57Q).

Durante su experiencia los futuros docentes comprendieron la importancia de trabajar la cultura en la clase de lengua extranjera, lo cual tiene que ver con lo señalado por Paige et al. (2000), quienes afirman que el lenguaje, en el proceso de enseñanza/ aprendizaje, desempeña un doble papel fascinante y 
complejo: es un medio y un modelador de cultura. Esto se logra al incluir el contexto de los niños, pero compartiendo con ellos sobre otros entornos y maneras de vivir en el mundo: "la clase de lengua debe basarse en el contexto de los alumnos pero, a su vez, en todos los aspectos que se viven en otros contextos (P59E)".

Al involucrar a sus estudiantes en ese diálogo relacional con respecto a ámbitos culturales diversos, los futuros maestros parecieron incrementar su comprensión del impacto que esta práctica pedagógica podría tener en la construcción de la identidad propia:

\begin{abstract}
Me di cuenta de que nuestra cultura puede fortalecerse creando una identidad si se les permite a los estudiantes establecer contactos con culturas de países muy lejanos [...] tuve que incentivar mi creatividad para llevarles a ellos clases en las que no solo aprendieran los contenidos, sino que además ampliaran su visión del mundo (P30Q).
\end{abstract}

\section{Conclusiones e implicaciones}

Una primera respuesta a la pregunta que guio esta investigación fue la revaloración que hicieron los futuros maestros de principios pedagógicos para la enseñanza de idiomas, aprendidos en el transcurso de su educación universitaria, lo cual concuerda con los estudios realizados en Colombia por Bonilla y Cruz-Arcila (2014) y Cruz-Arcila (2018). Los participantes entendieron el uso del español -en su práctica- como apoyo a la dinámica comunicativa que debe caracterizar el aprendizaje de una lengua extranjera. Así mismo, comprendieron que, más allá de una instrucción fundamentada en el léxico y la práctica oral, se deben involucrar procesos lectoescritores, e introducir prácticas y reflexiones que, si bien pueden retar la cultura local, también pueden traer opciones de vida beneficiosas a los estudiantes.

En segundo lugar, la cultura pedagógica de la escuela rural, mediada por el enfoque de la Escuela Nueva, fue asumida como un reto para integrar la enseñanza de los idiomas. En este sentido, a pesar de una percepción de dificultad para manejar aulas de alta diversidad entre sus integrantes, lo cual también es reportado en la investigación de Olleta (2013), los participantes expandieron sus horizontes respecto a la utilización del trabajo colaborativo y el valor del desarrollo de la autonomía del estudiante, como herramientas guías en estos contextos.

En tercer lugar, los participantes afianzaron estrategias y conocimientos que, desde la pedagogía general, se entrelazaron con la didáctica específica para lenguas extranjeras. Vieron la pertinencia de propuestas transversales que buscaban conectar el inglés con otras asignaturas, las cuales implicaban temáticas muy pertinentes al contexto rural, como también lo determinaron los estudios de Quintero (2016) y Zuluaga et al. (2009). Igualmente, fomentaron el encuentro de los ámbitos rurales y urbanos, y de las culturas: colombiana, anglosajona y francesa, implicando la conexión intrínseca entre lenguaje y cultura al educar en lengua extranjera y favorecer en sus estudiantes el desarrollo, no solamente de conocimientos sobre otras tradiciones, sino también de entendimiento y fortalecimiento de su propia identidad.

Por último, los participantes advirtieron la importancia que tiene la educación centrada en los perfiles de los estudiantes. En conexión directa con esto, el trabajo con infantes que tenían necesidades especiales se constituyó en un aspecto primordial de su aprendizaje. Los futuros maestros se enfrentaron a este reto auto-educándose, dada la falta de conocimientos en esta área y la imposibilidad de contar con acompañamiento especializado en este aspecto.

Teniendo en cuenta los anteriores hallazgos, se sugiere fortalecer el currículo de los programas de formación docente haciendo énfasis en perspectivas críticas que permeen las áreas de pedagogía, cultura y lenguaje, pues se observó cómo los futuros maestros, al apoyarse en los perfiles de sus estudiantes y la naturaleza de sus contextos, planteaban opciones pedagógicas más significativas tanto para ellos como para sus estudiantes. De igual manera, la integración de componentes en la formación pedagógica acerca del trabajo con estudiantes que presentan necesidades especiales 
y en contextos de alta diversidad, como el aula multigrado, debería convertirse en fundamento de programas que tienen influencia en contextos rurales, como los involucrados en este estudio,

Finalmente, se percibe la necesidad de fortalecer la perspectiva sociolingüística y sociocultural en el plan de estudios que cursan los futuros maestros. En particular, se ve la necesidad de resaltar el valor de las variedades de la lengua materna y de las costumbres y tradiciones propias de los contextos rurales, dado que estas no deberían ser rechazadas o mal vistas, pues reflejan la identidad y el origen de un grupo de personas que las utilizan y que constituyen una parte esencial de nuestra nación.

\section{Referencias}

Arriagada, M. (2015). L2 teaching strategies used by teachers in multigrade rural schools in Chile: a case study [Tesis doctoral, Universidad de Chile]. Repositorio institucional de la Universidad de Chile. http://repositorio.uchile.cl/handle/2250/132542

Berner-Rodoreda, A., Bärnighausen, T., Brinkmann, S., Eyal, N., Kennedy, C., McMahon, S. y Wikler, D. (2020). From doxastic to epistemic: a typology and critique of qualitative interview styles. Qualitative Inquiry, 26(3-4), 291-305. https://doi. org/10.1177/1077800418810724

Bonilla, S. y Cruz-Arcila, F. (2014). Critical socio-cultural elements of the intercultural endeavour of English teaching in Colombian rural areas. Profile Issues in Teachers' Professional Development, 16(2), 117133. http://dx.doi.org/10.15446/profile.v16n2.40423

Burns, A. y Richards, J. (2009). Introduction second language teacher education. En A. Burns y J. Richards (Eds.), The Cambridge Guide to Second Language Teacher Education (pp. 1-8). Cambridge University Press (CUP).

Byram, M. (1991). Teaching culture and language: towards an integrated model. En D. Buttjes y $M$. Byram (Eds.), Mediating languages and cultures: towards an intercultural theory of foreign language education (pp. 17-32). Multilingual Matters.

Chiu, M. M., Núñez, I. D., Pérez, B. M. y Mendoza, M. M. (mayo, 2013). La enseñanza del inglés a escuelas primarias rurales de Tapachula Chiapas, caso: escuela primaria Otilio Montaño [Ponencia]. Foro Internacional de Especialistas en Enseñanza de Lenguas Gregorio Torres Quintero, Manzanillo
(Colima), México. http://cenedic.ucol.mx/fieel/2013/ ponencias pdf/62.pdf

Cohen, L., Manion, L. y Morrison, K. (2018). Questionnaires. En L. Cohen, L. Manion y K. Morrison (Eds.), Research methods in education (pp. 471-505). Routledge.

Cruz-Arcila, F. (2018). The wisdom of teachers' personal theories: creative ELT practices from Colombian rural schools. Profile Issues in Teachers' Professional Development, 20(2), 65-78. https://doi. org/10.15446/profile.v20n2.67142

Dörnyei, Z. y Taguchi, T. (2009). Questionnaires in second language research: construction, administration, and processing. Routledge.

Freeman, D. (2002). The hidden side of the work: Teacher knowledge and learning to teach. A perspective from North American educational research on teacher education in English language teaching. Language teaching, 35(1), 1-13. https://doi.org/10.1017/ $\underline{\text { S0261444801001720 }}$

Freeman, D. y Johnson, K. E. (1998). Reconceptualizing the knowledge-base of language teacher education. TESOL Quarterly, 32(3), 397-417. https://doi.org/10.2307/3588114

Gil, N. y Ruiz, L. (2009). La enseñanza-aprendizaje del idioma inglés en las comunidades rurales y el enfoque comunicacional. Evaluación e Investigación, 4(2), 79-92.

Glesne, C. (2015). Becoming qualitative researchers: an introduction ( $5^{\mathrm{a}}$ ed.). Pearson.

Gómez, V. M. (2010). Una visión crítica sobre la Escuela Nueva de Colombia. Revista educación y pedagogía, 7(14-15), 280-306.

González, J. (2010). Los aspectos positivos y negativos de la educación en la escuela rural. Revista Digital Innovación y Experiencias Educativas, 30, 1-13.

Hawkins, M. (2004). Introduction. En M. Hawkins (Ed.), Language learning and teacher education: a sociocultural approach (pp. 3-9). Multilingual Matters.

Hittotuzi, N. (2006). The learners' mother tongue in the L2 learning-teaching symbiosis. Profile Issues in Teachers' Professional Development, 7(1), 161-171.

Johnson, K. E. (2009). Second Language Teacher Education: A Sociocultural Perspective. Routledge.

Kumaravadivelu, B. (2006). Understanding language teaching: from method to postmethod. Routledge.

Martínez, S., Pertuz, M. C. y Ramírez, J. M. (2016). La situación de la educación rural en Colombia, los desafíos del posconflicto y la transformación 
del campo. Compartir/FEDESARROLLO. https:// www.compartirpalabramaestra.org/documentos/ fedesarrollo compartir/la-situacion-de-la-educacionrural-en-colombia-los-desafios-del-posconflicto-y-latrasformacion-del-campo.pdf

Merriam, S. B. (2009). Qualitative research: a guide to design and implementation ( $2^{\mathrm{a}}$ ed.). Jossey-Bass.

Miles, M. B., Huberman, A. M. y Saldaña, J. (2014). Qualitative data analysis: a methods sourcebook (3 $3^{a}$ ed.). Sage.

Noguera, M. (2012). Fortalecimiento de la enseñanza del inglés en el Instituto Nacional Público Monseñor Víctor Manuel Soto Gutiérrez [Tesis de Maestría, Universidad de Zaragoza]. Repositorio institucional de la Universidad Nacional Autónoma de Nicaragua, León (RIUL). http://riul.unanleon.edu.ni:8080/jspui/ bitstream/123456789/369/1/220759.pdf

Olleta, L. (2013). La enseñanza de inglés en los centros rurales [Trabajo de Grado, Universidad de Valladolid]. Repositorio institucional de la Universidad de Valladolid. http://uvadoc.uva.es/handle/10324/3155

Ortega, D. y Fernández, R. A. (2017). La educación ecuatoriana eninglés: nivel de dominioy competencias lingüísticas de los estudiantes rurales. Revista Scientific, 2(6), 52-73. https://doi.org/10.29394/ scientific.issn.2542-2987.2017.2.6.3.52-73

Paige, R. M., Jorstad, H., Siaya, L., Klein, F. y Colby, J. (2000). Culture learning in language education: a review of the literature (Information Analyses 070). Minnesota University. https://files.eric.ed.gov/fulltext/ ED475523.pdf

Pérez, M. (2016). Currículo Transversal en la contemporaneidad. Escenarios, 14(1), 85-101. http://ojs.uac.edu.co/index.php/escenarios/article/ view/881

Perfetti, M. (2004). Estudio sobre la educación para la población rural en Colombia. FAO/UNESCO. https:// es.calameo.com/books/0062750482aa6cf738d42

Quintero, J. (2006). Contextos culturales en el aula de inglés. Íkala, 11(1), 151-177.

Ramos, B. y Aguirre, J. (2016). English language teaching in rural areas: a new challenge for English language teachers in Colombia. Cuadernos de Lingüística Hispánica, 27, 209-222. https://doi. org/10.19053/0121053X.4217
Rodríguez, M. y Otálora, E. (2018). Prácticas docentes en la enseñanza de inglés en educación básica en instituciones educativas públicas en contextos rurales [Trabajo de Grado, Universidad de La Salle]. Repositorio institucional de la Universidad de $\mathrm{La}$ Salle (Ciencia Unisalle). https://ciencia.lasalle.edu.co/ lic lenguas $/ 514 /$

Roldán, Á. M. y Peláez, O. A. (2017). Pertinencia de las políticas de inglés en zonas rurales colombianas: un estudio de caso en Antioquia. Íkala, 22(1), 121-139. https://doi.org/10.17533/udea.ikala.v22n01a08

Sanz del Río, S. (1991). La integración escolar. I. Evolución y realidad actual. Zerbitzuan, 15, 21-26.

Shulman, L. (1987). Knowledge and teaching: foundations of the new reform. Harvard Educational Review, 57(1), 1-23. https://doi.org/10.17763/ haer.57.1.j463w79r56455411

Singh, G. y Richards, J. C. (2006). Teaching and learning in the language teacher education course room: a critical sociocultural perspective. RELC Journal, 37(2), 149175. https://doi.org/10.1177/0033688206067426

Varón, S., Giraldo, M. y Gutiérrez, T. (2018). Tensiones entre el documento "Derechos básicos de aprendizaje de inglés: de transición a quinto de primaria" y la educación rural. [Trabajo de Grado, Universidad de La Salle]. Repositorio institucional de la Universidad de La Salle (Ciencia Unisalle). https://ciencia.lasalle.edu.co/cgi/viewcontent. cgi?article $=1508 E$ context $=$ lic lenguas

Viáfara, J. J. (2011). How do EFL student teachers face the challenge of using L2 in public school classrooms? Profile Issues in Teachers' Professional Development, 13(1), 55-74.

Yin, R. K. (2013). Case study research: design and methods (5 ed.). Sage.

Zamora, L., Vargas, M. y Rincón, A. (2012). Abordajes en la formación de educadores para el ejercicio rural de la docencia. Editorial Códice.

Zuluaga, T., López, M. y Quintero, J. (2009). Integrating the coffee culture with the teaching of English. Profile Issues in Teachers' Professional Development, 11(2), 27-42. 